\title{
Subacute Thyroiditis Developed While Waiting for Papillary Thyroid Cancer Surgery: Pathologically Proven Two Cases
}

\author{
Do-Hoon Kim ${ }^{1}$, Chae Moon Hong ${ }^{1}$, Man-Hoon $\mathrm{Han}^{2}$ and Jaetae Lee ${ }^{1}$ \\ Department of Nuclear Medicine, Kyungpook National University School of Medicine and Hospital ${ }^{1}$, Department of Pathology, \\ Kyungpook National University School of Medicine and Hospital ${ }^{2}$, Daegu, Korea
}

\begin{abstract}
Co-existence of subacute thyroiditis and papillary thyroid cancer (PTC) is rarely reported. We have recently experienced interesting cases of subacute thyroiditis, which developed while waiting for elective surgery of thyroid cancer in two patients. Two women, aged 52 and 55 years, suspected or diagnosed as PTC complained of anterior neck pain and febrile sensation several weeks before the scheduled surgery. Both cases showed elevated serum thyroid hormones and erythrocyte sedimentation rate, and decreased thyroid-stimulating hormone. III-defined hypoechoic lesions on ultrasonography and decreased uptake on Tc-99m pertechnetate scan were noted in both lobes. Total thyroidectomy for PTC was performed after relief of symptoms either by steroid or non-steroid anti-inflammatory drug treatment. Pathologic evaluation of surgical specimen revealed multinucleated giant cells and mononuclear cell infiltration. Pathognomic findings of subacute thyroiditis in addition to PTC were observed in both cases.
\end{abstract}

Key Words: Subacute thyroiditis, Papillary thyroid cancer, Thyroid nodule, Hypoechoic area

\section{Introduction}

Subacute thyroiditis (SAT), also known as subacute granulomatous or de Quervain thyroiditis, is the most common cause of thyroid pain. ${ }^{1,2)}$ Patients with SAT complain of anterior neck pain, commonly preceded by upper respiratory inflammation. ${ }^{1,2)}$ The incidence of thyroid cancer is increasing worldwide, probably due to the widespread use of sensitive diagnostic tools such as ultrasound (US). ${ }^{3)}$ Due to the fact that both SAT and thyroid cancer occur most frequently in middle-aged women, the presence of SAT and papillary carcinoma could be observed in the same subject. However, it is uncommon in the clinics, and patho- logical confirmed cases from surgical specimens have rarely been reported. Herein, we report two cases of the pathologically proven co-existence of SAT and papillary thyroid cancer (PTC) in two middle-aged women. Thyroid cancer was suspected by find needle aspiration cytology (FNAC), and elective total thyroidectomy was scheduled in both cases. Painful neck swelling and mild thyrotoxic symptoms were developed while waiting for surgery in both cases. Typical findings suggesting SAT and PTC were observed in both cases via microscopic evaluation of the surgical specimen acquired by total thyroidectomy.

Received October 12, 2021 / Revised October 25, 2021 / Accepted November 1, 2021

Correspondence: Jaetae Lee, MD, PhD, Department of Nuclear Medicine, Kyungpook National University School of Medicine and Hospital, 130 Dongdeok-ro, Jung-gu, Daegu 41944, Korea

Tel: 82-53-420-5586, Fax: 82-53-422-0864, E-mail: jaetae@knu.ac.kr

Copyright (c) the Korean Thyroid Association. All rights reserved.

(1) () This is an open-access article distributed under the terms of the Creative Commons Attribution Non-Commercial License (http://creative(c) (1) \& 8 commons.org/licenses/by-nc/4.0/), which permits unrestricted non-commercial use, distribution, and reproduction in any medium, provided the original work is properly cited. 


\section{Case Reports}

\section{Case 1}

A 52-year-old female patient was transferred to our clinic for a palpable nodule in the right lobe of the thyroid gland discovered during health screening. Thyroid US showed a $1 \times 1.2 \mathrm{~cm}$ hypoechoic nodule with scattered microcalcifications in the right upper lobe, which was confirmed as suspicious papillary thyroid carcinoma (Category IV according to the Bethesda classification of thyroid cytologic evaluation) by FNAC (Fig. $1 \mathrm{~A}$ ). There were also two benign-looking nodules (1 $\mathrm{cm}$ and $0.5 \mathrm{~cm}$ ) in the right lobe and a heterogenous echotexture in both lobes (Fig. 1A, B). Serum levels of thyroid hormone, titers of antithyroglobulin antibody (anti- $\mathrm{Tg} \mathrm{Ab}$ ), and antimicrosomal antibodies (AMA) were within normal limits.

While waiting for the scheduled surgery, the patient presented fatigue and progressing neck pain, not improved after taking acetaminophen at home. On physical examination, tenderness on the firmly enlarged thyroid gland, especially the left lobe of the thyroid, was noted, and she complained that the tender area had somewhat increased in size during the recent days. Laboratory tests showed an increased erythrocyte sedimentation rate (ESR) of $84 \mathrm{~mm} / \mathrm{h}$ (normal range: $0-20 \mathrm{~mm} / \mathrm{h}$ ), but a normal leucocyte count. The levels of free thyroxine (fT4) and thyroid-stimulating hormone (TSH) were $1.67 \mathrm{ng} / \mathrm{dl}$ (normal range, 0.89$1.80 \mathrm{ng} / \mathrm{dl}$ ) and $0.05 \mathrm{mIU} / \mathrm{L}$ (normal range, 0.3-4.0 $\mathrm{mIU} / \mathrm{L}$ ), respectively. Serum titer of either anti-Tg Ab or AMA was within normal limits. Tc-99m pertechnetate thyroid scan showed markedly decreased tracer uptake in both lobes of the thyroid, which was compatible to findings of SAT (Fig. 1D).

Oral prednisolone (10 mg per day) was prescribed and, as a result, most of her symptoms including the anterior neck pain were improved within a week and the tenderness on the thyroid gland was also resolved. Steroid was completely stopped at 1 month with disappearance of the symptoms and tenderness on physical examination. Follow-up laboratory test conducted at 2 months after symptom onset revealed a normal ESR of $16 \mathrm{~mm} / \mathrm{h}$ and normal leucocyte count,
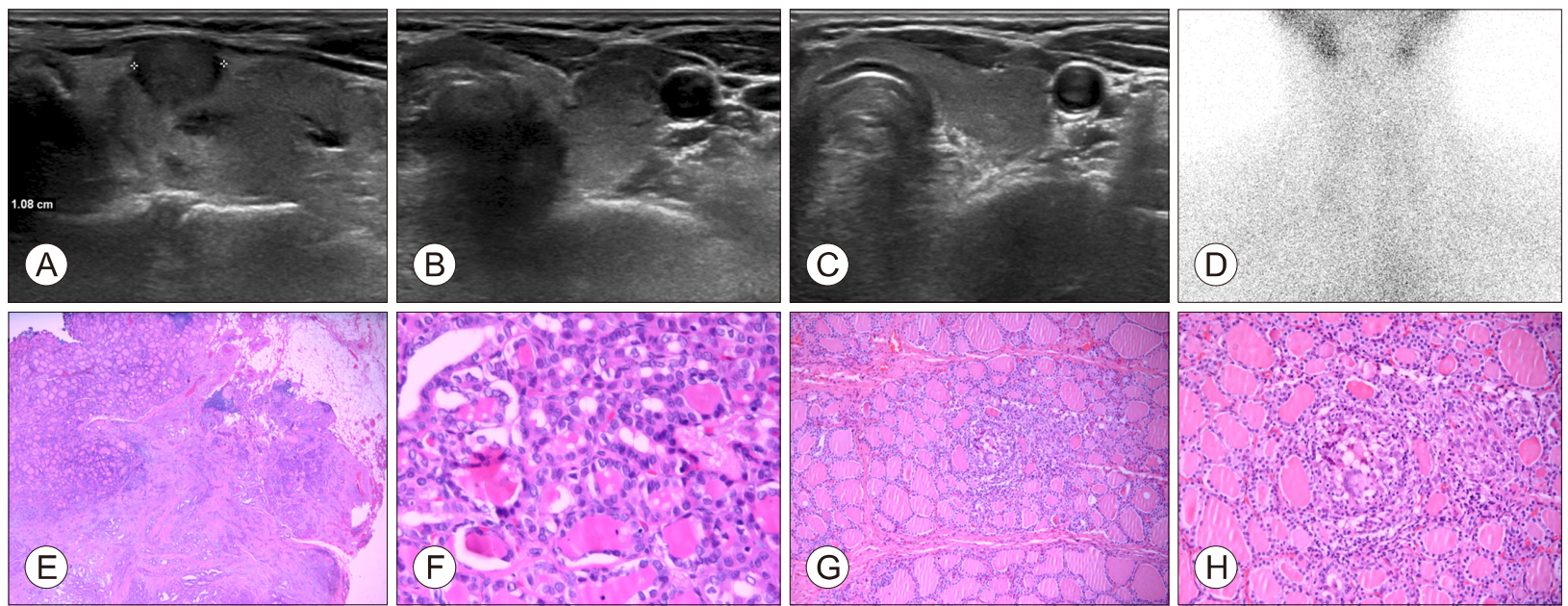

Fig. 1. Image and histopathological findings of patient 1. Longitudinal $(A)$ and transverse $(B, C)$ thyroid ultrasonographic images. Longitudinal image (A) shows a well-defined, $1.0 \times 1.2 \mathrm{~cm}$ sized hypoechoic, microcalcified nodule in the right lobe and strap muscle invasion of the nodule is suspected. In addition, diffuse hypoechogenicity was noted adjacent to the thyroid nodule. Transverse image (B) reveals an ill-defined hypoechoic area in the subcapsular area of the left lobe, findings compatible with thyroiditis. Follow-up transverse image (C) shows recovery from thyroiditis. Tc-99m pertechnetate thyroid scan (D) shows marked decreased tracer uptake in both lobes of the thyroid. (E) (H\&E stain, $\times 40)$ and $(F)(H \& E$ stain, $\times 200)$ show papillary carcinoma. Papillary carcinoma is associated with interstitial fibrosis that results from desmoplastic changes due to carcinoma. (G) (H\&E stain, $\times 100)$ and $(H)(H \& E$ stain, $\times 200)$ show granulomatous inflammation and interstitial fibrosis with multinucleated giant cells, which suggest subacute thyroiditis. 
and her thyroid hormone and TSH levels also became normal. Follow-up US showed almost complete disappearance of the hypoechoic lesion (Fig. 1C).

The patient underwent total thyroidectomy and right central neck node dissection, because mass was grossly extended to the surrounding soft tissue. Pathologic evaluation of the surgical specimen confirmed a $1.2-\mathrm{cm}$ papillary carcinoma with direct extension into the strap muscle and perithyroidal soft tissue. Lymphatic vascular or peri-neural invasion was absent, and all lymph nodes of the right central neck were negative for cancer involvement. She was diagnosed with T4aNO PTC based on the American Joint Committee on Cancer (AJCC) 8th edition, and microscopic evaluation of the non-cancerous area of the thyroid gland revealed scattered areas of granulomatous inflammation with the destruction of follicular epithelial cells and colonization of multinucleated giant cells, consistent with the classic features of SAT (Fig. 1E-H).

The patient was administered $100 \mathrm{mCi}$ of adjuvant radioactive iodine under recombinant human TSH stimulation, and subsequent whole body $1-131$ scin- tigraphy taken at 7 days revealed no iodine uptake in the thyroid bed. She has been free of clinical recurrence and her serum thyroglobulin level remained undetectable at six months after surgery.

\section{Case 2}

A 55-year-old woman presented vague neck discomfort and feelings of increased size of a known neck mass, which was improved with acetaminophen in the recent two weeks. Her medical record stated that a huge $5-\mathrm{cm}$ mass in the left lobe of the thyroid gland was observed on US 5 years ago at a local hospital. Neck computed tomography also demonstrated a large well-demarcated low-dense nodule in the corresponding region and an ill-defined nodular lesion in the right lobe (Fig. 2A). Repeated FNAC tests showed atypia of undetermined significance (AUS) two times, but in two other tests, it showed a benign follicular lesion (Fig. 2B); however, she refused further visits despite the recommendation for surgical excision after the last FNAC performed 2 years prior.

She had experienced flu-like symptoms before the visiting our hospital. Thyroid US revealed a $5.0-\mathrm{cm}$
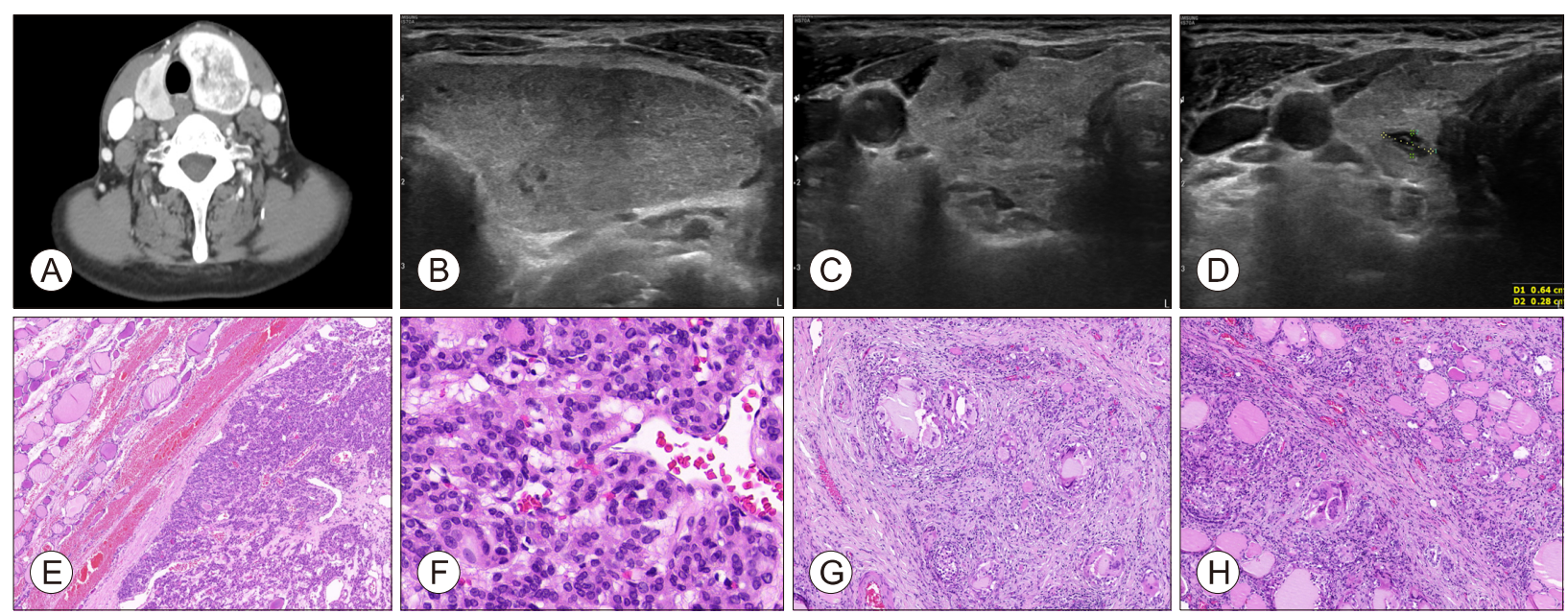

Fig. 2. Image and histopathological findings of patient 2. Neck CT (A) show a large well-demarcated low-dense nodule in the left lobe. In addition, the transverse image of neck ultrasonography (B) shows a large heterogeneous hypoechoic nodule in the left lobe, which was confirmed as a follicular variant of papillary carcinoma after surgery. Transverse image (C) shows diffuse ill-defined hypoechogenicity in the subcapsular area; it looks taller than wide and has a speculated margin. FNAC was performed at the hypoechoic nodule, and the result showed AUS. In the follow-up ultrasonographic image (D), the hypoechoic nodule has significantly and the subcapsular diffuse hypoechogenicity has improved. (E) (H\&E stain, $\times 40)$ and $(F)(H \& E$ stain, $\times 400)$ show papillary carcinoma with encapsulated follicular variant. $(G)(H \& E$ stain, $\times 100)$ and $(H)(H \& E$ stain, $\times 100)$ show granulomatous inflammation and interstitial fibrosis with multinucleated giant cells, which suggest subacute thyroiditis. 
heterogenous echogenic nodule in the left lobe, which was not quite different from images taken two years ago. There also noted an irregular-margined deep hypoechoic lesion in the right lobe (Fig. 2C). FNAC for lesions in both lobes were undertaken and results were AUS for both lesions, while cells of the right lesion were rather suggesting medullary thyroid cancer. Laboratory evaluation showed mildly increased ESR of $23 \mathrm{~mm} / \mathrm{h}$ and a subclinical hyperthyroid state. Serum levels of fT4 was $1.64 \mathrm{ng} / \mathrm{dl}$, with a low TSH level of $0.18 \mathrm{mIU} / \mathrm{L}$. Both anti-Tg Ab and AMA titers were normal. Serum levels of calcitonin and carcinoembryonic antigen were also normal.

Follow-up US study taken two weeks later demonstrated no changes in findings for the left nodular lesion, while changes in shape and decrease in size of the hypoechoic lesion was observed in the right lobe, suggesting a benign nature (Fig. 2D). Her laboratory findings including ESR, fT4, and TSH were within normal limits.

Elective total thyroidectomy and both central neck dissection were performed one month after the visit. Pathologic evaluation of the surgical specimen revealed a 5.0-cm-sized follicular variant of papillary carcinoma in the left lobe. While in the right lobe, scattered areas of granulomatous inflammation and multinucleated giant cells were noted, which are typical findings compatible to those of the recovery phase of SAT (Fig. 2E-H). There was no cancer lesion in the resected lymph nodes acquired by central neck dissection. She was diagnosed as T3aNO based on the AJCC 8th edition.

\section{Discussion}

We have presented two cases showing pathologically proven SAT and PTC in the same subject. Our cases were unique, particularly because symptoms and signs of SAT developed while waiting for an elective surgery of PTC. The second patient developed symptoms suggesting SAT, but did recognize that was attributed to the enlargement of a preexisting thyroid nodule. Diagnosis of SAT and PTC was confirmed by microscopic evaluation of surgical specimen after thy- roidectomy in both cases.

SAT is a spontaneously remitting inflammatory disorder, possibly due to a post-viral response. ${ }^{1,2)}$ The peak age of incidence is 40 to 50 years. ${ }^{4)}$ It is suggested that there is a seasonal peak, but this is not always confirmed. ${ }^{4)}$ Clinically, neck pain is a typical manifestation of SAT, and there are also common symptoms associated with thyroid dysfunction. ${ }^{5)}$ On palpation, the thyroid gland is painful, tender, and enlarged. Systemic symptoms include fever, fatigue, muscle pain, and anorexia. ${ }^{6,7)}$ Thyroid dysfunction usually follows a tri-phasic course (thyrotoxicosis, hypothyroidism, and euthyroidism) that usually lasts 3 months. ${ }^{5)}$ Patients usually return to euthyroidism within 6 to 12 months. ${ }^{8)}$ However, permanent hypothyroidism develops in $5 \%$ to $31 \%$ of patients, requiring long-term replacement with levothyroxine. ${ }^{9}$

The diagnosis of SAT is usually based on clinical evidence, but laboratory tests and imaging studies are useful. ${ }^{6,710)}$ Significant elevations in inflammatory markers (ESR and C-reactive protein) are common. ${ }^{5}$ In the acute phase, most individuals have overt thyrotoxicosis (high fT4 levels and low or undetectable TSH levels). ${ }^{6)}$ As a result of the destruction process, the free triiodothyronine (fT3)/fT4 ratio is usually low and the thyroglobulin level is high. ${ }^{6,7)}$ TSH receptor antibodies are usually absent, whereas AMA is positive in some patients and anti-Tg Ab is positive in a larger number of patients. ${ }^{11,12)}$ During the acute phase, thyroid scan showed reduced uptake, as observed in case 1 , and decreased RAIU. ${ }^{10)}$

In the biopsy, granulomatous infiltrate, sometimes with giant cells and consistent with a viral infection, is commonly observed. ${ }^{9)}$ Mononuclear cell infiltration occurs first, followed by the appearance of multinucleated giant cells and necrosis later, which are common features in the inflammatory process of viral infection in tissues. Given that surgery was performed after improvement of the clinical manifestation of the SAT, we believe that mild-to-moderate mononuclear cell infiltration and scattered multinucleated giant cells were present in our cases.

SAT requires treatment for painful conditions and symptoms of thyrotoxicosis. ${ }^{13)}$ Beta-blockers are used 
to control thyrotoxic symptoms and anti-inflammatory therapy for pain control. ${ }^{13)}$ The American Thyroid Association's clinical guidelines recommend non-steroidal anti-inflammatory drugs (NSAIDs) for mild symptoms and $40 \mathrm{mg} /$ day prednisolone for patients with severe disease or those who do not respond to NSAID treatment. ${ }^{14)}$ Acute symptoms were well controlled with steroid or NSAID in our cases and surgery was successfully undertaken two months after initial symptoms.

Differentiated thyroid cancer arises from follicular cells of the thyroid gland, and accounts for almost all thyroid cancers, and PTC accounts for approximately $85 \%$ of differentiated thyroid cancers. ${ }^{15)}$ US is the most important imaging modality in differentiating malignant from benign nodules since US can detect thyroid nodules in $19-68 \%$ of randomly selected individuals. ${ }^{16)}$ It has been reported that the bilateral hypoechoic areas with low to absent vascularization commonly observed in SAT is sometimes misinterpreted as PTC lesion. ${ }^{10,17)}$ The hypoechoic lesion on US is produced by the inflammatory process associated with viral infections. Nishihara et al. ${ }^{8)}$ reported that if the extent of hypoechoic lesion is large and bilateral, the development of hypothyroidism is very likely to occur after SAT. Of 252 patients with SAT, 15 patients (5.9\%) developed permanent hypothyroidism, and bilateral hypoechogenic areas of the thyroid were present in the initial examination despite any treatment groups. ${ }^{8)}$

Given that both PTC and SAT are rare thyroid disorders, there is also a possibility of the co-occurrence of these two pathologic lesions in the same subject. However, there are not many reports indicating the co-occurrence of SAT and PTC in the same subject. Sung et al. ${ }^{3)}$ previously reported a rare case of SAT with coexisting PTC, which was suspected incidentally by US. Nishihara et al. ${ }^{18)}$ reported that five patients with PTC were detected in 1152 Japanese patients with SAT. While a study of 160 SAT patients from Minnesota, USA, demonstrated no presence of thyroid cancer among them. ${ }^{9}$ In contrast, in a recent study of 137 (100 females) patients confirmed as SAT by histopathologic evaluation of the excised thyroid, six patients (4.4\%) also had PTC. ${ }^{19)}$ Those were micro- carcinomas in most of cases. The US findings of markedly hypoechoic lesions with irregular margins of SAT renders the differential diagnosis of PTC more difficult. ${ }^{13,17)}$ This may sometimes be due to the masking of the small PTC located at or adjacent to extensive hypoechoic lesions of the SAT. ${ }^{13,17)}$ Conversely, unnecessary surgery in patients with SAT can be avoided by careful review of early clinical-US findings and performing US-FNA or follow-up US. ${ }^{17)}$

In summary, we report pathologically proven cases of coexisting SAT and thyroid papillary carcinoma in the same subject. Our report suggests that SAT lesions can also be detected during preoperative staging work-up of thyroid cancer. Thus, combined US and laboratory test is helpful for the differential diagnosis of SAT in cases developing symptoms possibly related to SAT.

\section{Acknowledgments}

We are grateful to the staff of Kyungpook National University School of Medicine and Hospital for their valuable contribution.

\section{Authorship Contribution Statement}

Do-Hoon Kim, Chae Moon Hong, Man-Hoon Han, and Jaetae Lee participated in data collection and data interpretation. Do-Hoon Kim and Jaetae Lee wrote and edited the manuscript. Do-Hoon Kim, Chae Moon Hong, and Jaetae Lee contributed to study design, reviewed and edited the manuscript.

\section{Conflicts of Interest}

No potential conflict of interest relevant to this article was reported.

\section{Funding Statement}

This research received no specific grant from any funding agency in the public, commercial, or not-forprofit sectors. 


\section{Orcid}

Do-Hoon Kim: https://orcid.org/0000-0003-3703-9796

Chae Moon Hong: https://orcid.org/0000-0002-5519-6982

Man-Hoon Han: https://orcid.org/0000-0001-8856-553X

Jaetae Lee: https://orcid.org/0000-0001-5676-4059

\section{References}

1) Pearce EN, Farwell AP, Braverman LE. Thyroiditis. $N$ Engl J Med 2003;348(26):2646-55.

2) Bindra A, Braunstein GD. Thyroiditis. Am Fam Physician 2006;73(10):1769-76.

3) Sung PS, Kim MH, Lim DJ, Choi YH, Kang MI, Cha BY, et al. Subacute thyroiditis with coexisting papillary carcinoma. J Korean Thyroid Assoc 2011;4(1):58-62.

4) Samuels MH. Subacute, silent, and postpartum thyroiditis. Med Clin North Am 2012;96(2):223-33.

5) Brancatella A, Ricci D, Viola N, Sgro D, Santini F, Latrofa F. Subacute thyroiditis after SARS-CoV-2 infection. J Clin Endocrinol Metab 2020;105(7):dgaa276.

6) Nishihara E, Ohye H, Amino N, Takata K, Arishima T, Kudo T, et al. Clinical characteristics of 852 patients with subacute thyroiditis before treatment. Intern Med 2008;47(8): 725-9.

7) Benbassat CA, Olchovsky D, Tsvetov G, Shimon I. Subacute thyroiditis: clinical characteristics and treatment outcome in fifty-six consecutive patients diagnosed between 1999 and 2005. J Endocrinol Invest 2007;30(8):631-5.

8) Nishihara E, Amino N, Ohye H, Ota H, Ito M, Kubota S, et al. Extent of hypoechogenic area in the thyroid is related with thyroid dysfunction after subacute thyroiditis. J Endocrinol Invest 2009;32(1):33-6.

9) Fatourechi V, Aniszewski JP, Fatourechi GZ, Atkinson EJ, Jacobsen SJ. Clinical features and outcome of subacute thyroiditis in an incidence cohort: Olmsted County, Minnesota, study. J Clin Endocrinol Metab 2003;88(5):2100-5.
10) Bennedbaek FN, Hegedus L. The value of ultrasonography in the diagnosis and follow-up of subacute thyroiditis. Thyroid 1997;7(1):45-50.

11) Nishihara E, Amino N, Kudo T, Kohsaka K, Ito M, Fukata $\mathrm{S}$, et al. Moderate frequency of anti-thyroglobulin antibodies in the early phase of subacute thyroiditis. Eur Thyroid J 2019; 8(5):268-72.

12) Ricci D, Brancatella A, Marino M, Rotondi M, Chiovato L, Vitti $\mathrm{P}$, et al. The detection of serum IgMs to thyroglobulin in subacute thyroiditis suggests a protective role of IgMs in thyroid autoimmunity. J Clin Endocrinol Metab 2020;105(6):dgaa038.

13) Sencar ME, Calapkulu M, Sakiz D, Hepsen S, Kus A, Akhanli $\mathrm{P}$, et al. An evaluation of the results of the steroid and non-steroidal anti-inflammatory drug treatments in subacute thyroiditis in relation to persistent hypothyroidism and recurrence. Sci Rep 2019;9(1):16899.

14) Ross DS, Burch HB, Cooper DS, Greenlee MC, Laurberg P, Maia AL, et al. 2016 American Thyroid Association guidelines for diagnosis and management of hyperthyroidism and other causes of thyrotoxicosis. Thyroid 2016;26(10):1343-421.

15) Aschebrook-Kilfoy B, Ward MH, Sabra MM, Devesa SS. Thyroid cancer incidence patterns in the United States by histologic type, 1992-2006. Thyroid 2011;21(2):125-34.

16) Haugen BR, Alexander EK, Bible KC, Doherty GM, Mandel SJ, Nikiforov YE, et al. 2015 American Thyroid Association management guidelines for adult patients with thyroid nodules and differentiated thyroid cancer: the American Thyroid Association guidelines task force on thyroid nodules and differentiated thyroid cancer. Thyroid 2016;26(1):1-133.

17) Park HK, Kim DW, Lee YJ, Ha TK, Kim DH, Bae SK, et al. Suspicious sonographic and cytological findings in patients with subacute thyroiditis: two case reports. Diagn Cytopathol 2015;43(5):399-402.

18) Nishihara E, Hirokawa M, Ohye H, Ito M, Kubota S, Fukata $\mathrm{S}$, et al. Papillary carcinoma obscured by complication with subacute thyroiditis: sequential ultrasonographic and histopathological findings in five cases. Thyroid 2008;18(11):1221-5.

19) Gul N, Uzum AK, Selcukbiricik OS, Yegen G, Tanakol R, Aral F. Prevalence of papillary thyroid cancer in subacute thyroiditis patients may be higher than it is presumed: retrospective analysis of 137 patients. Radiol Oncol 2018;52(3):257-62. 